\title{
Is Prevalence of Hypertension Increasing in First-Ever Stroke Patients?: A Hospital-Based Cross-Sectional Study
}

\author{
Abhishek Pathak ${ }^{\mathrm{a}}$ Pradeep Kumar $^{\mathrm{b}}$ Awadh Kishor Pandit ${ }^{\mathrm{b}}$ \\ Kamalesh Chakravarty ${ }^{b}$ Shubham Misra ${ }^{b}$ Arun Kumar Yadav ${ }^{b}$ \\ Kameshwar Prasad ${ }^{b}$ \\ ${ }^{a}$ Department of Neurology, All India Institute of Medical Sciences, New Delhi, India; ${ }^{\mathrm{b}}$ Institute of Medical Sciences, \\ Banaras Hindu University, Varanasi, India
}

\section{Keywords}

Hypertension · Stroke - Cardiovascular disease - Cerebral infarction · Risk factor $\cdot$ Registries $\cdot$ Stroke epidemiology

\begin{abstract}
Background: Stroke is a devastating and disabling cerebrovascular disease with some amount of residual deficit leading to economic loss. Recent Indian studies have shown a stroke prevalence rate of $471.58 / 100,000$ populations. Hypertension has been known to be the main risk factor for causing stroke. Purpose: To investigate the prevalence of hypertension in first-ever stroke patients and its comparison with the previous stroke registry. Methods: The study was a hospital-based cross-sectional study. Consecutive patients, who were admitted in the Neurology ward of All India Institute of Medical Sciences (AIIMS), New Delhi, India, were recruited for the study from the period July 2012 to January 2014. The stroke units consisted of a computerized record containing the details of all the admitted patients. Results: A total of 260 patients were recruited in which 194 (74.6\%) were ischemic and 66 (25.4\%) were hemorrhagic stroke patients. Hypertension was present in 169 (65\%) patients. When compared with the previous stroke registry of 2,628
\end{abstract}

\section{KARGER}

() 2018 S.Karger AG, Basel

E-Mail karger@karger.com

www.karger.com/aon patients, hypertension was recorded in 1,503 (57.2\%) patients. Conclusion: Our data show that there is an increase in the proportion of hypertension among first-ever stroke patients reported in AlIMS in the years 2012-2014 as compared to that reported in during the period 1998-2011.

(c) 2018 S.Karger AG, Basel

\section{Introduction}

Stroke is a major public health problem and the second leading cause of death and long-term disability all over the world [1]. Stroke has accounted for nearly 5.7 million deaths worldwide; $87 \%$ of these deaths occur in low- and middle-income countries [2]. About $85 \%$ of stroke cases are ischemic, while the remaining $15 \%$ are hemorrhagic. This has an enormous socioeconomic impact on the patients, their families, and health services. The incidence of stroke in South Asian countries has increased by more than $100 \%$, while it is decreased by $42 \%$ in developed European countries in the past 4 decades [3]. South Asian population is at an increased risk of stroke owing to the environmental and genetic risk factors including hypertension, diabetes, smoking, dyslipidemia, atrial fibrilla- 
tion, family history of stroke, low socioeconomic status, and low education $[4,5]$. Risk factor assessment is an important step toward understanding the origin of a disease and in formulating a preventive strategy. Despite various guidelines laid down for primary prevention, the risk factor prevention is very low [6]. Several community- and hospital-based studies have identified hypertension as the most important risk factor for stroke. It has been hypothesized that stroke patients admitted to the All India Institute of Medical Sciences (AIIMS), New Delhi, recently have a higher prevalence of hypertension as compared to those admitted before. This hypothesis was chosen keeping in mind that the cost-effective drug treatments for hypertension, hypercholesterolemia, and atrial fibrillation reduced stroke risk, heart disease, and mortality [7]. This hypothesis was based on the observations made by a senior neurologist. Hence, this study was carried out to ascertain the prevalence of hypertension in first-ever stroke patients admitted to the Neurology ward of AIIMS.

\section{Methods}

The study was conducted in the Department of Neurology, AIIMS, New Delhi. The study was a hospital-based, cross-sectional study and was completed in one and half years (July 2012 to January 2014). Patients with a history of transient ischemic attack, fever, rheumatologic disease, autoimmune disease, any acute or chronic infection, and a history of regular immunosuppressive or analgesic therapies were excluded. A total of 194 ischemic stroke (IS) and 66 hemorrhagic stroke (HS) patients were recruited for the study after radiologic confirmation of stroke by $\mathrm{CT}$ or MRI scans of the brain.

All patients had clinical signs consistent with the World Health Organization definition of stroke. Written informed consent was obtained from all patients before the collection of information and blood samples. The study was approved by the local Institutional Ethics Committee.

\section{Clinical Examination}

A detailed history and clinical evaluation was carried out. IS was classified using the Trial of Org 10172 in Acute Stroke Treatment criteria [8]. The National Institute of Health Stroke Scale, modified Rankin Scale, and Barthel Index scores were used for determining clinical severity and independency.

\section{Definition of Variables}

Hypertension: subjects will be considered to have hypertension if they either have the diagnosis of hypertension or treated for hypertension before the stroke or reference date. In addition, if a control will have no recorded blood pressure before the reference date but diastolic pressure of $90 \mathrm{~mm} \mathrm{Hg}$ or more or a systolic pressure of $140 \mathrm{~mm} \mathrm{Hg}$ or more on 2 or more occasions during the study evaluation, he or she will be considered to have hypertension. Dyslipidemia: if they either were diagnosed with dyslipidemia or were treated for dyslipidemia before the stroke or reference date. Diabetes: if a subject will have the diagnosis document- ed by a physician on the medical record or if fasting blood sugar level will be $>126 \mathrm{mg} / \mathrm{dL}$. Smoker: a regular smoker was defined as a person smoking $\geq 1$ cigarettes, bidis, and cigar daily for more than 3 preceding months. Family history of stroke: a positive family history of stroke was diagnosed if a subject had a first-degree relative (parent or sibling) who had had a stroke. Hypercholesterolemia: it was defined by a total cholesterol level of $220 \mathrm{mg} / \mathrm{dL}$ or if the patient was under lipid-lowering therapy. We also recorded alcohol intake, smoking, atrial fibrillation, history of transient ischemic attack, previous myocardial infarction, and peripheral vascular disease.

\section{Statistical Analysis}

Data analysis was done by using Statistical Program for Social Sciences (SPSS) for windows version 17 (SPSS Inc., Chicago, IL, USA). Categorical variables were expressed as frequency and percentage, while continuous variables were expressed as mean and standard deviation. Students $t$ test was used for continuous and normally distributed data. Chi-square test was used to compare frequency distributions between 2 or more groups. $p$ value $<0.05$ was considered statistically significant.

\section{Results}

Two hundred-sixty patients were admitted in the inward of the neurology department comprising 200 (76.9\%) males. The mean age was 50.3 years. Diabetes was present in 60 (25.2\%). Hypertension was present in 169 (65\%). History of alcohol intake was present in 64 $(25.2 \%)$. The baseline characteristics are explained in Table 1.

The distribution of Trial of Org 10172 in Acute Stroke Treatment subtypes of IS patients has been diagrammatically presented in Figure 1. Among the 194 IS patients, 59 (29.2\%) had large vessel disease, 35 (17.3\%) had small vessel disease, 51 (25.2\%) had Cardioembolic stroke, 36 (17.8\%) had negative evaluation, and 20 (9.9\%) had other undetermined etiology.

We also tried to investigate the history of hypertension and divided the patients into whether already diagnosed as hypertensive, diagnosed at the time of admission and not known and found that $6.1 \%$ of the patients were diagnosed at the time of admission, while $68 \%$ were diagnosed at the time of admission and of all hypertensive patients, $70 \%$ of the patients were not taking any drugs at the time of admission for the IPD patients. There were a total of 240 deaths in the old registry in comparison to 55 deaths in the present registry. The number of deaths in ischemic and hemorrhagic groups were 187 and 53 in the previous registry and $23(4.7 \%)$ and $32(24 \%)$ in the present registry respectively. 
Fig. 1. Distribution of ischemic stroke based on TOAST classification for R2 Registry. TOAST, Trial of Org 10172 in Acute Stroke Treatment; LVD, large vessel disease; SVD, small vessel disease.

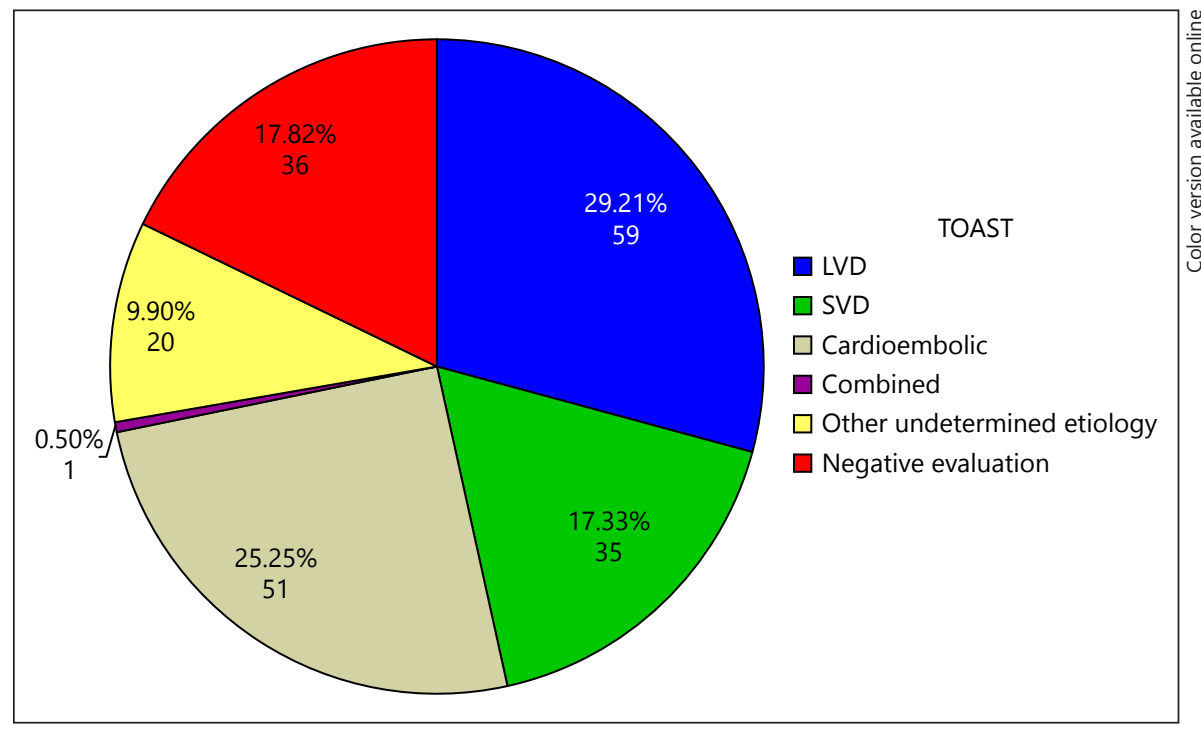

Table 1. Baseline characteristics of patients presenting with stroke

\begin{tabular}{lccc}
\hline Continuous variables, mean $\pm \mathrm{SD}$ & $\begin{array}{c}1998-2011(\mathrm{R}=1) \\
(n=2,628)\end{array}$ & $\begin{array}{l}2012-2014(\mathrm{R}=2) \\
(n=260)\end{array}$ & $p$ value \\
\hline Age, years & $55.15 \pm 15.01$ & $50.33 \pm 14.23$ & $<0.001$ \\
Systolic blood pressure & $151.16 \pm 33.73$ & 0.0015 \\
Diastolic blood pressure & $90.98 \pm 18.57$ & 0.008 \\
Total cholesterol, mmol/L & $122.81 \pm 90.84$ & $87.73 \pm 15.89$ & $<0.001$ \\
Triglycerides, mmol/L & $99.68 \pm 95.08$ & $171.52 \pm 110.14$ & $<0.001$ \\
HDL cholesterol, mmol/L & $48.15 \pm 14.46$ & $129.97 \pm 69.54$ & $<0.001$ \\
LDL cholesterol, mmol/L & $77.09 \pm 56.45$ & $41.69 \pm 9.04$ & $<0.001$ \\
Glucose, mmol/L & $69.62 \pm 73.21$ & $96.43 \pm 35.83$ & 0.001 \\
Gender, male, $n(\%)$ & $1,587(65.4)$ & $124.79 \pm 47.64$ & 0.21 \\
Hypertension, $n(\%)$ & $1,503(57.2)$ & $200(76.9)$ & 0.88 \\
Smoking, $n(\%)$ & $921(35)$ & $169(65)$ & 0.85 \\
Alcohol intake, $n(\%)$ & $630(24.6)$ & $64(24.7)$ & 0.59 \\
Diabetes mellitus, $n(\%)$ & $560(21.3)$ & $60(25.2)$ & $\mathbf{0 . 0 0 0 2}$ \\
Myocardial infarction, $n(\%)$ & $190(7.2)$ & $38(14.6)$ & 0.80 \\
Ischemic, $n$ (\%) & $2,131(81.1)$ & $64(74.6)$ & $\mathbf{0 . 0 4}$ \\
Hemorrhagic, $n(\%)$ & $497(18.9)$ & $66(25.4)$ & \\
\end{tabular}

IPD, Inpatient Department; HDL, High Density Lipoprotein; LDL, Low Density Lipoprotein; ICH, Intracerebral Hemorrhage.

* Chi-square test for categorical variables and $t$ test for continuous variables.

\section{Discussion}

The presence of hypertension in HS patients was significantly higher in the current registry (R2) than the earlier registry (R1; Table 2$)$. The average age of onset of stroke admitted to the Neurology ward was significantly reduced in the R2 than in R1. A study published by Heuschmann et al. [9] in the South London stroke register showed age and hypertension as causes of stroke over a period of 10 years (1995-2004) and found that the median age over these 10 years decreased significantly from 73 to 71 years $(p=0.017)$. Correspondingly, hypertension as a cause of HS decreased from $72 \%$ in the year $1995-1996$ to $65.89 \%$ in the year $2003-$ 2004 ( $p=0.0036)$. Though our study was a hospitalbased study and cannot be externally validated to the population, the observations from the North Kentucky Stroke Study revealed that the mean age of stroke has decreased from $62.8 \pm 16.4$ to $59.4 \pm 17.9$ years $(p=$ 0.0003 ) in a survey conducted in 1995, while hyperten- 
Table 2. Proportion of the presence of hypertension differences in both the registries in ICH stroke patients in IPD patients

\begin{tabular}{lll}
\hline $1998-2012$ & $\begin{array}{l}2012-2014 \\
(\mathrm{R} 1), n(\%)\end{array}$ & $\begin{array}{l}\text { R1 (IPD) vs. R2 (IPD) } \\
(p \text { value })\end{array}$ \\
\hline $114(55.9)$ & $43(75.4)$ & $\mathbf{0 . 0 0 8}$ \\
\hline
\end{tabular}

sion as a cause of HS has increased from 39 to $59 \%$ in 2005 ( $p \leq 0.0001)$ [10]. Another study, observed the changes in the incidence and etiology of HS from a period of 1981 to 2006 in Oxfordshire, UK. The results indicated that there was a substantial fall in the hypertension associated with HS over the study period of the last 25 years. This trend was seen in patients $\leq 75$ years of age [11]. Béjot et al. [12] studied the intracerebral hemorrhage profiles of the patient in Dijon, France, in a population-based study from the period between 1985 and 2008 and found that the incidence rate remained almost unchanged over the period; however, the prevalence of hypertension increased from 55 to $70 \%$ but was statistically insignificant.

There are a few limitations to the present study. First, the study is done at the tertiary care center in North India and the results cannot be extrapolated to the community and could just represent the patients with the referral bias. Second, the study shows the presence of admission bias, as only sick patients requiring urgent admission and medical attention were admitted. Third, inadequate data were present for 130 patients and thus were excluded, while 30 patients died within hospital stay and were excluded from inclusion.
The average age of HS has decreased. Hypertension as an all-cause factor of HS has increased along with the percentage of population who refuse to take medication despite being hypertensive.

\section{Conclusion}

Our data show that there is an increase in the proportion of hypertension among first-ever stroke patients reported in AIIMS in the year 2012-2014 as compared to those reported in the year 1998-2011.

\section{Acknowledgment}

None.

\section{Disclosure Statement}

There was no conflict of interest among the authors.

\section{Funding Source}

No funding source.

\section{Author Contribution}

Contributor AP has collected data and written the final manuscript. P.K. and A.K.Y. supervised the statistical analysis. A.K.P. helped in screening patients and collecting samples. S.M. and K.C. helped in designing and writing the manuscript. K.P. helped in developing the concept and designing the protocol and corrected the manuscript to the final version.

\section{References}

1 Feigin VL, LawesCM, BennettDA, et al: Worldwide stroke incidence and early case fatality reported in 56 population-based studies: a systematic review. Lancet Neurol 2009;8:355-369.

2 Strong K, Mathers C, Bonita R: Preventing stroke: saving lives around the world. Lancet Neurol 2007;6:182-187.

3 Feigin VL: Stroke epidemiology in the developing world. Lancet 2005;365:2160-2161.

4 Wang PL, Zhao XQ, Yang ZH, et al: Effect of in-hospital medical complications on case fatality post-acute ischemic stroke: data from the China National Stroke Registry. Chin Med J (Engl) 2012;125:2449-2454.

5 Carrera E, Maeder-Ingvar M, Rossetti AO, et al: Trends in risk factors, patterns and causes in hospitalized strokes over 25 years: the Lau- sanne Stroke Registry. Cerebrovasc Dis Basel Switz 2007;24:97-103.

6 Kearney PM, Whelton M, Reynolds K, et al: Worldwide prevalence of hypertension: a systematic review. J Hypertens 2004;22:11-19.

7 Wright JM, Musini VM, Gill R: First-line drugs for hypertension. Cochrane Database Syst Rev 2018;4:CD001841.

8 Adams HP Jr, Bendixen BH, Kappelle LJ, et al: Classification of subtype of acute ischemic stroke. Definitions for use in a multicenter clinical trial. TOAST. Trial of Org 10172 in Acute Stroke Treatment. Stroke 1993;24:3541.

9 Heuschmann PU, Grieve AP, Toschke AM, et al: Ethnic group disparities in 10-year trends in stroke incidence and vascular risk factors: the South London Stroke Register (SLSR). Stroke 2008;39:2204-2210.

10 Kleindorfer DO, Khoury J, Moomaw CJ, et al: Stroke incidence is decreasing in whites but not in blacks: a population-based estimate of temporal trends in stroke incidence from the Greater Cincinnati/Northern Kentucky Stroke Study. Stroke 2010;41:1326-1331.

11 Lovelock CE, Molyneux AJ, Rothwell PM: Change in incidence and aetiology of intracerebral haemorrhage in Oxfordshire, UK, between 1981 and 2006: a population-based study. Lancet Neurol 2007;6:487-493.

12 Béjot Y, Cordonnier C, Durier J, et al: Intracerebral haemorrhage profiles are changing: results from the Dijon population-based study. Brain 2013;136:658-664. 